\title{
Compatibility of Two System of Field-Aligned Currents
}

\author{
P.A. Sedykh \\ Irkutsk National Research Technical University., Irkutsk, Russian Federation \\ E mail (pvlsd@mail.ru).
}

\section{Accepted : 2 September 2019}

\begin{abstract}
The combined action of convection and pitch-angle diffusion of electrons and protons is responsible for the formation of gas pressure distribution in the magnetosphere. Plasma pressure, in turn, determines - within the framework of a given magnetic field model - the density of bulk currents in the magnetosphere. With knowledge of the bulk currents as a function of coordinates, we can calculate the field-aligned currents as a divergence of bulk currents. On the other hand, specifying the convection model is equivalent to specifying the electric field model. Since within the approximation of equipotential field lines the electric field is common to the magnetosphere and ionosphere, bulk currents and fieldaligned currents in the ionosphere can be formally calculated subject to the condition that ionospheric conductivity is wholly determined by electron precipitation from the magnetosphere. Sun illumination is important for dayside region. The precipitation intensity is readily inferred from our structurally adequate model of the geomagnetosphere. Thus, we have two systems of field-aligned currents. One system is calculated from the model of plasma pressure distribution in the magnetosphere, and the other is inferred from a given model of the electric field and the electroconductivity model calculated from electron precipitation. This brings up the question: How can these two systems of field-aligned currents be reconciled? From previous studies it is known that magnetospheric convection "adjusts itself" to the level of energy losses in the ionosphere. Based on this, an attempt can be made to achieve a compatibility of the aforementioned two systems of field-aligned currents. This paper is devoted to analyzing such an attempt.
\end{abstract}

(c) 2019 BBSCS RN SWS. All rights reserved

Keywords: Field-Aligned Currents,

\section{Introduction}

The problem of magnetosphere-ionosphere coupling is very interesting. Magnetospheric Alfvén waves are reflected by the ionosphere. According to (Ellis, Southwood, 1983), the fieldaligned currents (FACs) in the subsidiary Alfvén waves serve to close divergent horizontal currents resulting from the non-uniform ionospheric conductivity.

According to [Zhu, et al., 1993), the inhomogeneity of ionospheric conductivity can lead to FACs, which originate in the ionosphere. Increasing the conductivity gradient (in the direction which is perpendicular to the incident wave field), can increase the ratio of the Hall and Pedersen conductivities, and lowering the conductivity all lead to a larger rotation of the reflected wave field (in the range of from a few up to $40^{\circ}$ ).

According to (Yoshikawa, et al., 2013a), the polarization charge produced by the Pedersen current divergence has a role to cancel and intensify the ambient background electric field inside and outside the high-conductivity band, respectively. The polarization charge produced by the Hall current divergence has a role to rotate the electric field from the background electric field, which causes a meandering of ionospheric convection flow along the boundary of a high-conductivity band are always perpendicular to each other. The Hall and Pedersen currents never close each other when conductances are homogeneous, but they can do that at the conductivity gradient region.

Authors of (Yoshikawa et al., 2013b) presented the formulation of the coupling between the ionospheric horizontal currents and FACs via shear Alfven waves, which can describe the formation of a Cowling channel without any a prior parameterization of the secondary (Hall polarization) electric field strength. They showed that the reflected wave can carry FACs that connect to divergent Hall currents. They identified how large the secondary electric field becomes, how efficiently the divergent Hall current is closed within the ionosphere, and how much of the Hall current continues out to the magnetosphere as FACs. Authors concluded that only a small fraction of FACs is connected to Hall currents at conductance gradients.

The ionosphere is an ohmic environment, where the electric field and the current are related by the Ohm's law. If the ionospheric current were purely Hall currents, this would not be a dangerous phenomenon since the Hall current is nondivergent and does not deliver a work. In fact, the ionospheric current is combined and always includes the Pedersen component, and the ionosphere is a real energy consumer (sink). It is known that the contents of the magnetic flux tube to be referred to as the plasma tube throughout the text, transfers from one magnetic flux tube to another in the convection process without surplus and deficiency in the case where the field lines of the magnetic flux tube are equipotential ones. This idealization is quite realistic everywhere apart from polar auroras.

Then, as the plasma tube drifting toward the Earth in a dipole field, its volume decreases in proportion to $\mathrm{L}^{-4}$, and the situation is the reverse for density, while pressure increases in proportion to $\sim \mathrm{L}^{20 / 3}$. However, the process of adiabatic compression is attended by the processes of plasma tube depletion due to pitch-angle diffusion into the loss cone. This process is described by the factor $\sim \exp \left(-\int \mathrm{d} t / \tau\right)=\exp \left(-\int \mathrm{dr} / \mathrm{V}_{\mathrm{r}} \tau\right)=\exp \left(-\int \mathrm{rd} v / \mathrm{V}_{v} \tau\right)$ (Ponomarev, 1985). Thus gas pressure has a maximum on each line of convection. In accordance with the equation for $p_{\mathrm{g}}$ (Ponomarev, 1985; Ponomarev, Sedykh, 2006), we have:

$$
p_{g}=p_{g}^{0}\left(\frac{L_{\infty}}{L}\right)^{\frac{20}{3}} \exp \left(-\frac{5}{3} \int \frac{d r}{V_{r} \tau}\right)
$$

It is evident that $\mathrm{dt}=\mathrm{dR} / \mathrm{V}_{\mathrm{R}}=\mathrm{R}_{0} \mathrm{~d} \lambda / \mathrm{V}_{\lambda}, \Delta \mathrm{t}=\int \mathrm{dt}$ is the transport time, i.e., the time over which the flux tube will move from the boundary to the given point on the flux line; and $V_{R}$ and $V_{\lambda}$ are the radial and azimuthal components of convection velocity; $\mathrm{L}$ is the McIlwain parameter. Thus (1.1) indicates how gas pressure changes when plasma moves along the convection line at a velocity $\mathrm{V}=\left(\mathrm{V}_{\mathrm{R}}{ }^{2}+\mathrm{V}_{\lambda}{ }^{2}\right)^{1 / 2} ; \tau$ - is a time over which the plasma tube loses $1 / \mathrm{e}$ part or the initial number of particles $\tau=\tau_{0} \mathrm{~L}^{4}\left(\tau_{0}=1 \mathrm{~s}\right.$ for electrons, $\tau_{0}=12-18 \mathrm{~s}$ for protons). The radial components of convection velocity is $\mathrm{V}_{\mathrm{r}}=\mathrm{V}_{0} \mathrm{~L}^{9 / 2} \cos \lambda$. The azimuthal components of convection velocity is $\mathrm{V}_{\lambda}=-2.5 \mathrm{~V}_{0} \mathrm{~L}^{9 / 2} \sin \lambda+\mathrm{V}_{1} \mathrm{~L} ; \mathrm{V}_{1}$ - is a function of initial values of energy of electrons and protons; $\mathrm{V}_{0}=\mathrm{c}[\mathrm{E} \times \mathrm{B}] / \mathrm{B}^{2} ; \quad \gamma$ is the adiabatic exponent; $p_{\mathrm{g}}^{0}$ is an initial pressure at the boundary $L=L_{\infty}$ for particles with the corresponding energy. 

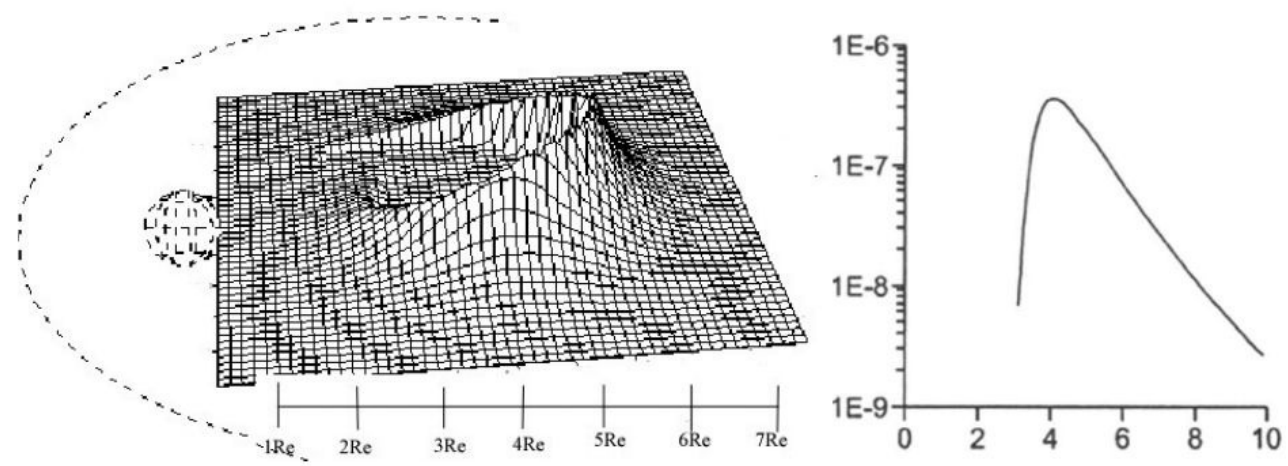

Fig. 1. Results of modeling. Plasma pressure distribution for steady-state boundary conditions. The profile (calculated values) of plasma pressure: $P,\left[\mathrm{dyn} / \mathrm{cm}^{2}\right] / L\left(R_{\mathrm{E}}\right)$

The pitch-angle diffusion of particles into the loss cone, together with adiabatic compression of plasma during the convection into the magnetosphere determines the behavior of the contents of the magnetic flux tube, the plasma tube. Gas pressure builds up under the action of an adiabatic compression of plasma drifting in a magnetic field with increasing strength and precipitation-induced losses.

In this case the magnetosphere develops a plasma pressure distribution, such as shown in Fig. 1.

A gas pressure pattern has the form of an amphitheater with a clearly pronounced maximum near the midnight meridian, and with a sharp earthward "break". This "break" received the name "Inner Edge of the Plasma Sheet", IEPS.

In these calculations, boundary conditions are timeindependent, that is, the supply of plasma through the boundary remains uniform, and only the convection velocity changes. The pressure peak increases with the increasing electric field. Noteworthy are the relatively gentle backward slope of the relief and a very steep forward slope. If the boundary conditions are unsteady and pressure increases for a certain time on the boundary of the region, from which plasma starts, the resulting plasma "bunch" drifts downstream of the convection.

Partially, these results were obtained in the classical work by C.F. Kennel (1969), who studied in detail the process of pitchangle diffusion of electrons into the loss cone and estimated the actual value of $\tau_{\mathrm{e}}$. Kennel did not estimate the time of pitch-angle diffusion of protons $\left(\tau_{\mathrm{p}}\right)$, but he proposed a simple principle for determining this time. In an ultimate case, which was called pitchangle diffusion by Kennel, the loss cone should be filled during one bounce period of a particle with an average energy.

The situation changes principally when the boundary conditions are dependent on time. The soltion structure is so that $\mathrm{p}_{0}(\mathrm{t})$ can be considered as an input signal multiplied by the transfer function A.

Input parameters for modeling are the electric field of magnetospheric convection and the magnetic field in the magnetosphere. The function for the electric field variation can be applied from our paper (Ponomarev et al., 2006), taking into account the well-known paper (Volland, 1975). Our obtained result is also standard. The character of electric field distribution over the dawn-dusk meridian (Ponomarev et al., 2006) quite corresponds to the classical distribution obtained in (Heppner, 1977). We applied the dipole like magnetic field model, but with special additional parameters for stretching magnetotail. For illustration of results of modeling, we applied the following expressions. The expression (1.1) can be written in the form (Sedykh, Ponomarev, 2012):

$$
\mathrm{p}\left(\mathrm{t}^{\prime}\right)=\mathrm{G}(\mathrm{t}) \mathrm{A}(\mathrm{L})
$$

$$
\begin{aligned}
& E_{0}=E_{00}\left\{\begin{array}{l}
1 ; \quad t<-900 s \\
2 t / 1800+2 ; \quad-900 s \leq t \leq 900 s \\
1 ; \quad t>5400 s
\end{array}\right. \\
& G=G_{0}^{a}\left\{\begin{array}{l}
1 ; \quad t^{\prime}<0 s \\
2 t^{\prime} / 500+1 ; 0 s \leq t^{\prime} \leq 500 s \\
-t^{\prime} / 500+4 ; \quad 500 s<t^{\prime} \leq 1500 s \\
1 ; \quad t^{\prime}>1500 s
\end{array}\right.
\end{aligned}
$$

The expressions (1.3) are simple, and represent plasma pressure inhomogeneity in the magnetosphere during periods of magnetospheric disturbance development.

Since the relationship between the coordinate and time $(\mathrm{L}=$ $\int \mathrm{V}_{\mathrm{R}} \mathrm{dt}$ ) exists for the drifting plasma tube, the $\mathrm{p}_{0}$ disturbance can be represented as a disturbance moving with convection stream, the amplitude of which increases by a factor of A depending on L. If $\mathrm{p}_{0}$ and $\mathrm{A}(\mathrm{L})$ are narrow functions, their product is always close to zero except the instant when these functions coincide.

Since the resulting pressure amplitude is the product of the undisturbed signal amplitude by the magnitude of the disturbance, there emerges the picture shown in Fig. 2. The sequence in Fig.2 illustrates the pressure relief time history as a function of the convection electric field and nonstationary boundary conditions. One can see that at the time when the plasma bunch reaches the region of maximum steady-state pressure, there occurs a powerful short-duration outlier of plasma pressure (density and, accordingly, intensity of particle precipitation). We interpret it as break-up substorms (Sedykh, Ponomarev, 2012).

Since in plasma with isotropic pressure (within $3.5<\mathrm{L}<7$, where $\mathrm{L}$ is the Mcllwain parameter) the plasma pressure relief wholly determines the density of bulk currents for particles with the energy less than $15 \mathrm{keV}$, then:

$$
\mathbf{j}_{\perp}=\mathrm{c}\left[\mathbf{B} \times \nabla \mathrm{p}_{\mathrm{g}}\right] / \mathrm{B}^{2},
$$

where $\mathbf{B}$ is the magnetic field strength, $\mathbf{p}_{\mathrm{g}}$ is gas pressure, and $\mathbf{c}$ is the velocity of light.

\section{Formulation of the problem and computational technique}

The flux density of precipitating particles is rather sharply localized in the space and produces in the ionosphere a clearly pronounced precipitation oval (Fig. 3).

Sharp spatially localized regions of increased conductivity in the ionosphere also correspond to such a distribution of precipitation. In the energy range $1-20 \mathrm{keV}$ in which auroral electrons exist, about 30 electron-volt of the flux energy supplied to the ionosphere are expended in producing an electron-ion pair. 
A

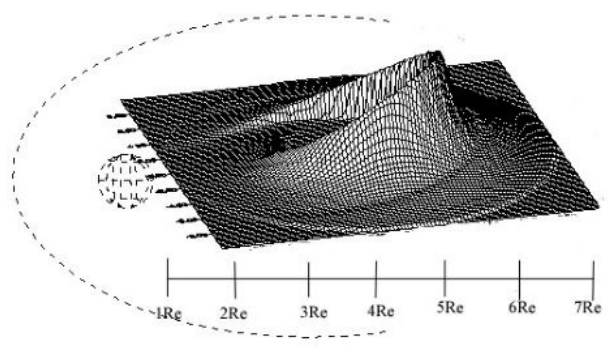

C

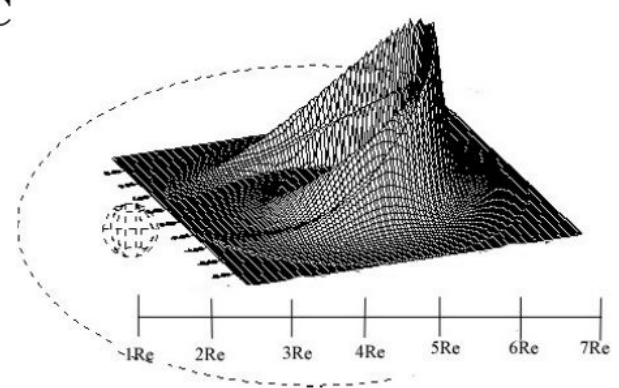

B

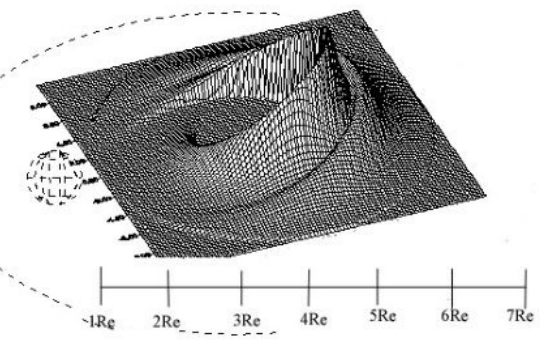

D

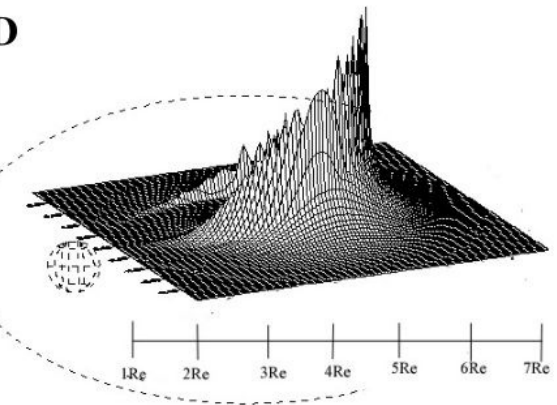

Fig. 2. The plasma pressure relief (results of modeling) under the nonstationary boundary conditions, and with the electric field which is variable in time (periods of magnetospheric disturbances): (a) $t=0 \mathrm{~s}$, (b) $t=1000 \mathrm{~s}$, (c) $t=2800 \mathrm{~s}$, (d) $t=4500 \mathrm{~s}$.

I

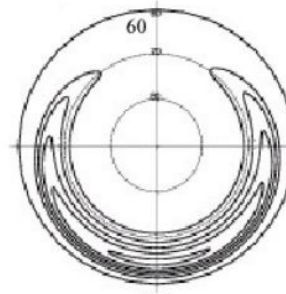

oh

a)

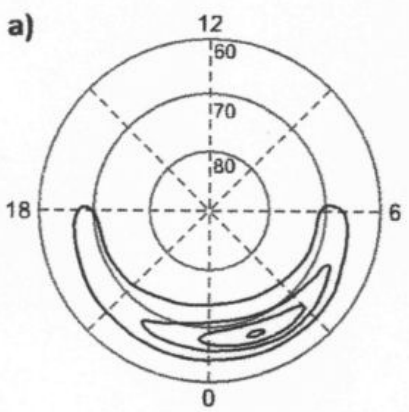

d)

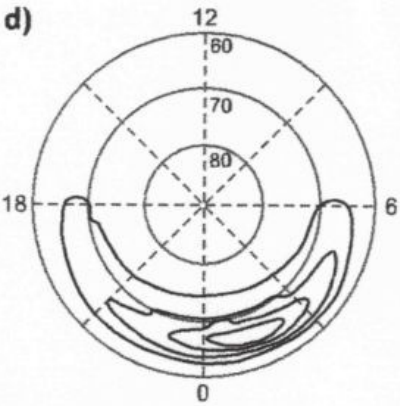

II

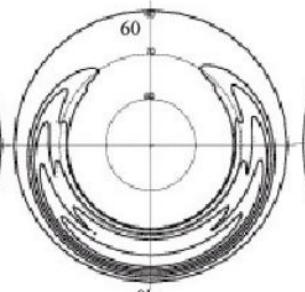

oh
III

b)

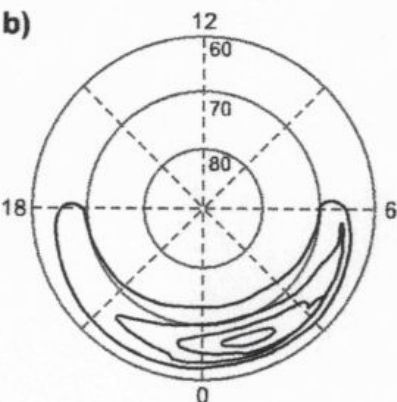

e)

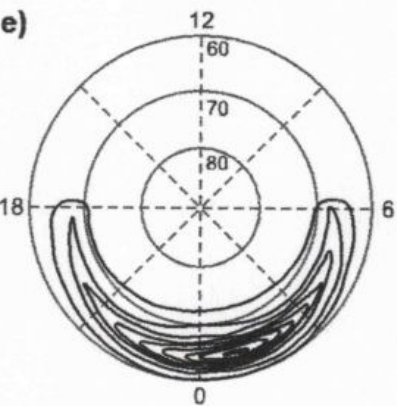

IV

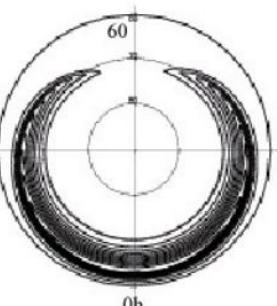

oh

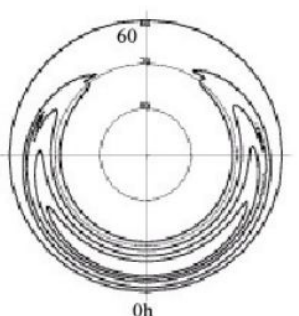

oh

c)

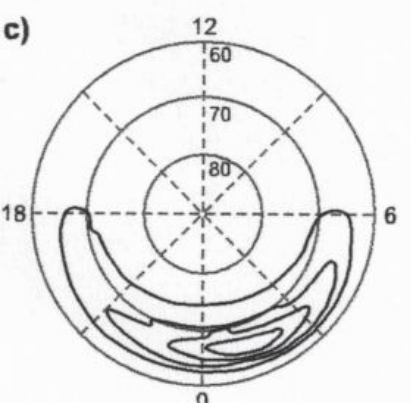

f)

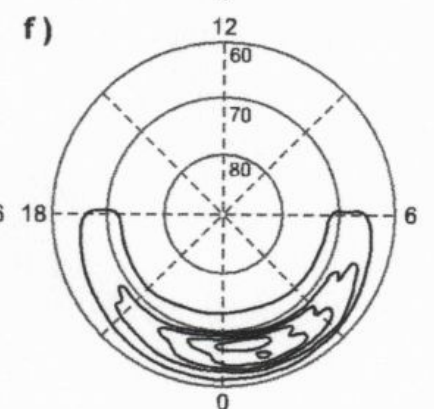

Fig. 3. Equidensity contours of the precipitating electron flux for unsteady boundary conditions: (I) $t=0 \mathrm{~s}$, (II) $t=1000 \mathrm{~s}$, (III) $t=2800 \mathrm{~s}$, and (IV) $t=4500 \mathrm{~s}$. More detailed pictures are for dusk side; results of modeling: contour lines of the intensity of the precipitating electron flux density for the nonstationary boundary conditions- a) $t 1=0 \mathrm{~s}, \mathrm{~b}) t 2=500 \mathrm{~s}, \mathrm{c}) t 3=1000 \mathrm{~s}, \mathrm{~d}) t 4=1800 \mathrm{~s}$, e) $t 5=2800 \mathrm{~s}, \mathrm{f}) t 6=4500 \mathrm{~s}$. 
Hence the ionization rate is $\sim \mathrm{j}_{\varepsilon} / \mathrm{H} \delta \varepsilon$ ( $\delta \varepsilon$ in ergs), where $\mathrm{j}_{\varepsilon}$ is the energy flux density of precipitating electrons (ergs/cm), and the electron density in steady-state conditions:

$$
\mathrm{n}_{\mathrm{e}} \sim\left(\mathrm{j}_{\varepsilon} / \mathrm{H} \delta \varepsilon \alpha\right)^{1 / 2}
$$

here $\delta \varepsilon$ is expressed in $\mathrm{erg} \mathrm{cm}^{-2}$; recombination coefficient $(\alpha)$, in $\mathrm{cm}^{3} \mathrm{~s}^{-1}$; and $H$ (the dynamo layer thick-ness), in $\mathrm{cm}$.

Integral conductivity for the Pedersen current is:

$$
\Sigma_{\mathrm{p}}=\left(\mathrm{e}^{2} \mathrm{n}_{\mathrm{e}} / \mathrm{M}_{\mathrm{i}}\right) \int v_{\mathrm{in}} /\left(\omega_{\mathrm{iB}}{ }^{2}+v_{\mathrm{in}}{ }^{2}\right) \mathrm{dz},
$$

where $\mathbf{e}$ is the electron charge, $\mathbf{M}_{\mathrm{i}}$ is the ion mass, $\omega_{\mathrm{iB}}$ is the ion gyrofrequency, and $\boldsymbol{v}_{\text {in }}$ is the ion-neutral collision frequency. The integral $(\mathrm{dz})$ is taken over the entire thick-ness of the currentcarrying layer, i.e., from 100 to $120 \mathrm{~km}$. Observational data and theoretical estimates show that the scale of the electric field along the latitude is several times larger than the scale of precipitation and, hence, than the scale of the conductivity region.

Since the ionization latitudinal distribution is much more nonuniform in space than the electric field, we will consider that:

$$
\partial J_{\theta} / \partial \theta \approx E_{\theta} \partial \Sigma / \partial \theta \text {. }
$$

$$
\partial \mathrm{J} / \partial \theta=\left(\partial \Sigma_{\mathrm{p}} / \partial \theta\right) \mathrm{E}_{\theta}+\left(\partial \mathrm{E}_{\theta} / \partial \theta\right) \Sigma_{\mathrm{p}} \sim \mathrm{E}_{\theta} \partial \Sigma_{\mathrm{p}} / \partial \theta
$$

Hence it follows that the electric field configuration is unimportant for the problem of generation of field-aligned currents in the ionosphere, at least for the divergence of those Pedersen currents, which flow along the latitude and produce "curtain" structures. Of importance are the parameters of precipitation, the intensity of which is closely associated with the spatial distribution of the number density of particles in the magnetosphere and, hence, with the pressure relief. For that reason, there must be a correspondence between the picture of field-aligned currents calculated from the gas pressure distribution in the magnetosphere and the picture of field-aligned currents calculated from the distribution of ionization (i.e. precipitation!).
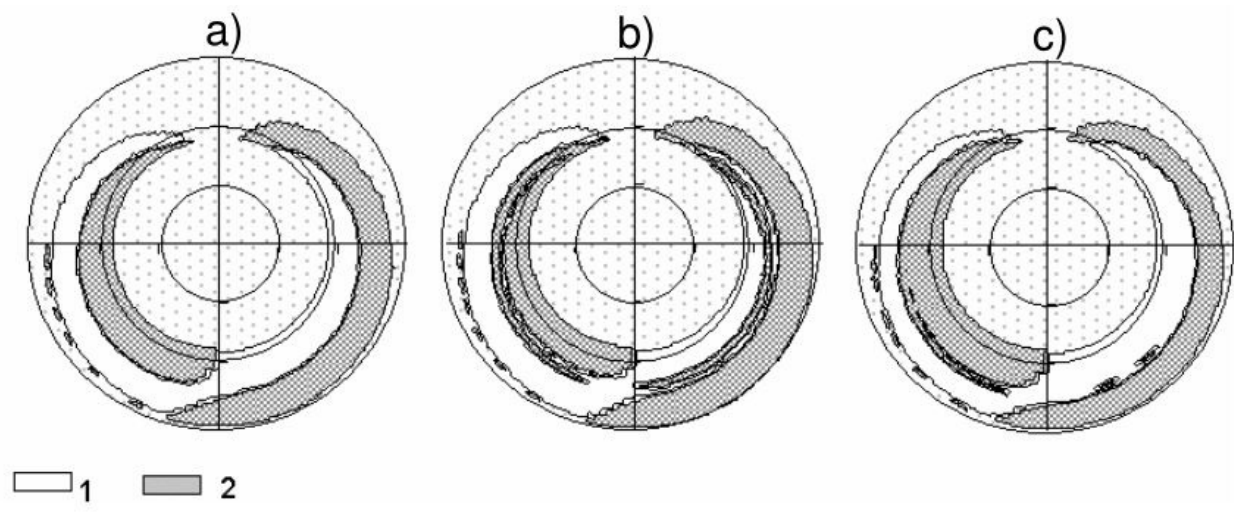

Fig. 4. Field-aligned currents "generated" in the ionosphere: 1 - zone of inflow currents, 2 - zone of outflow currents; a) $t=0 \mathrm{~s}$; b) $t=1000 \mathrm{~s}$; c) $\mathrm{t}=2800 \mathrm{~s}$.
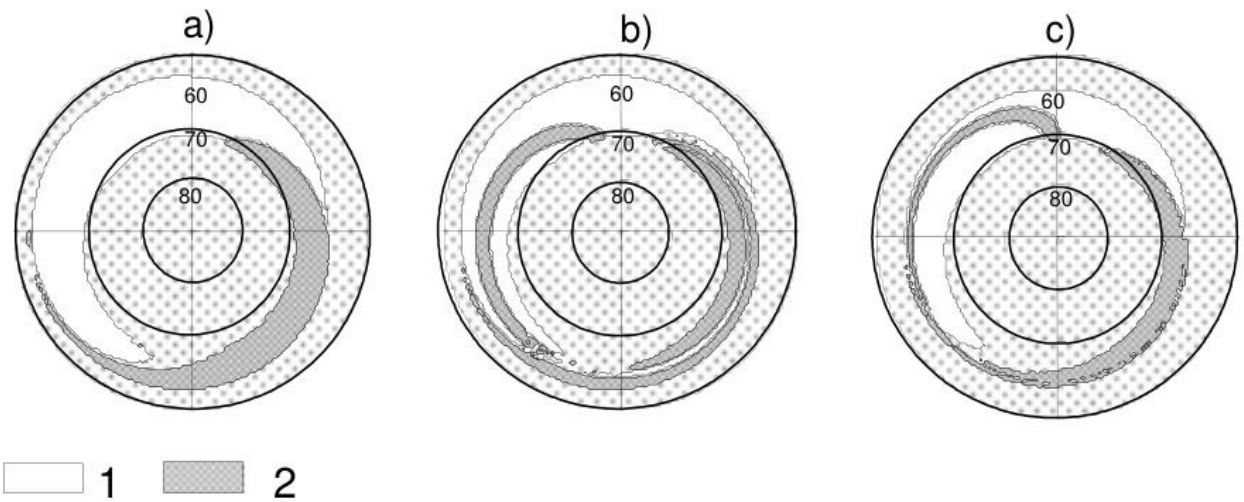

Fig.5. Field-aligned currents generated in the magnetosphere: I - zone of inflow currents, 2 - zone of outflow currents; a) $\mathrm{t}=0 \mathrm{~s}$; b) $\mathrm{t}=1000 \mathrm{~s}$; c) $\mathrm{t}=2800 \mathrm{~s}$. 1970; Ponomarev, 1985):
It is this reasoning that dictated the formulation of the problem. At the first stage of research, the result of which is presented in this paper, we present the pictures of field-aligned current distribution inferred in terms of a very simple model.

Calculations were performed by the formula:

$$
\mathrm{j}_{\Uparrow \mathrm{T}} \approx \mathrm{j}_{\mathrm{r}}=\left[\partial \mathrm{J}_{\lambda} / \partial \lambda+\cos \theta_{\mathrm{a}} \mathrm{J}_{\theta}+\sin \theta_{\mathrm{a}} \partial \mathrm{J} / \partial \theta\right] / \mathrm{r}_{\mathrm{o}} \sin \theta_{\mathrm{a}}
$$

Since in high latitudes the direction of geomagnetic field lines along the latitude, $\mathrm{J}_{\lambda}$, and along the meridian, $\mathrm{J}_{\theta}$, were calculated by standard formulas:

$$
\mathrm{J}_{\lambda}=\Sigma_{\mathrm{p}} \mathrm{E}_{\lambda} \quad \text { and } \quad \mathrm{J}_{\theta}=\Sigma_{\mathrm{p}} \mathrm{E}_{\theta}
$$

Results of calculations of field-aligned currents "generated" in the ionosphere are shown in Fig. 4.

It should be noted that only the sign of the field-aligned mplitude exceeded some given value, was plotted. For comparison, for the same instants of time Fig. 5 presents the signs of field-aligned currents generated in the magnetosphere.

Calculations were performed by the formula (Vasyliunas,

$\mathrm{j}_{\mathrm{ll}}=\mathrm{cB}^{\mathrm{I}} \int_{0}^{l}\left\{\left[\boldsymbol{\nabla} \mathbf{p}_{\mathrm{g}} \times \boldsymbol{\nabla} \mathbf{p}_{\mathbf{B}}\right] \cdot \mathbf{B} / \mathrm{p}_{\mathrm{B}} \mathrm{B}^{3}\right\} \mathrm{dl}$

where $\mathrm{B}^{\mathrm{I}}$ is the magnetic field strength in the ionosphere, the integral is taken over the entire flux tube from the equator to the ionosphere, and $\mathrm{p}_{\mathrm{B}}$ is the magnetic pressure. 


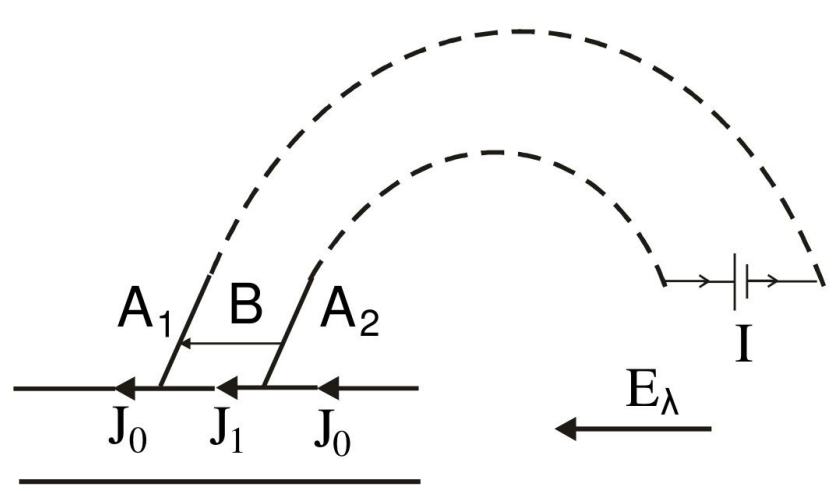

Fig.6. A - ionospheric region with background (low) conductivity. $B$ - ionospheric region with increased conductivity. The portion $A_{1}-A_{2}$ has a large-scale (quasi-homogeneous) meridional electric field $\mathrm{E}_{\lambda}^{\mathrm{A}}$ that produces the electric current. If the source of current I in the corresponding magnetospheric region is absent, then the current $j$ flows in the entire portion $A_{1}-A_{2}$; however, the electric field in region $B, E_{\lambda}^{B}$, is decreased. If, however, the source of current $I$ is present in the magnetosphere, then $E_{\lambda}$ is everywhere identical, and on portion $B$ the electric current $j^{B}$ is enhanced. Hence in the former case $j^{B}=j_{0}$ and $E_{\lambda}^{B}<E_{\lambda}^{A}$, and in the latter case $E_{\lambda}^{B}=E_{\lambda}^{A}$, but $j^{B}=j_{0}$.

It is clear that the integrand in (2.6) is proportional to the sine of the angle between the contour lines $\mathrm{p}_{\mathrm{g}}=$ const and $\mathrm{p}_{\mathrm{B}}=$ const. In a dipole approximation, lines of equal magnetic pressure are concentric circles, and isobars follow plasma pressure relief contour lines.

\section{Discussion of results}

The problem of compatibility of field-aligned currents generated in the magnetosphere, and of field-aligned currents, which are produced as a result of a spatial inhomogeneity of conductivity (and to a lesser extent, of the electric field), that is, as if they were "generated" in the ionosphere, is part of the problem of ionosphere-magnetosphere coupling. It is clear that in actual fact they are simply parts of one and the same global ionosphericmagnetospheric current system. The problem of ionospheremagnetosphere coupling primarily implies that it is necessary to solve the question as to how the magnetospheric producer of current and power "adjusts itself" to the ionospheric consumer. For a certain special configuration, this problem was solved in (Sedykh, Ponomarev, 2002; Ponomarev, Sedykh, Urbanovich, 2006; Sedykh, 2011; Sedykh, 2014).

It turned out, firstly, that the ionospheric consumer updates the convection rate and through it the plasma pressure gradient, which determines the density of bulk currents which, in turn, determines the behavior of field-aligned currents through its divergence. Secondly, it turned out that ionospheric and magnetospheric currents are not rigidly linked. Some of the current (and power!) that is not "demanded" by the ionosphere can go into feeding the MHD compressor pumping plasma into the region of increase magnetic pressure - in the earthward direction.

For us, the most important issue in this paper is that of ascertaining the direction of the cause-and-effect relationship. Current is primary in the magnetosphere, whereas the electric field is primary in the magnetosphere. Furthermore, the convection system can undergo some adjustment, and together with it the electric field in the ionosphere. But such adjustment is possibly only as corrections of the first approximation to the zero-order approximation. And hence the zero-order approximation, that is, the picture of field-aligned currents obtained essentially for an arbitrary but smooth initial electric field must contain the main elements of the natural system of field-aligned currents which is determine by the distribution of electron precipitation closely associated with the plasma pressure distribution in the magnetosphere. Let us now consider from this standpoint Figs. 4 and 5.

Fig. 4 shows a classical picture of field-aligned currents that coincides in its main traits with the well-known Iijima-Potemra scheme (Iijima, Potemra, 1978). This correspondence indicates that the factor that determines the main features of the configuration of field-aligned currents is the existence in the ionosphere of a well conducting channel produced by zones of intense precipitation of electrons from the plasma pressure hump region (see Figs. 1-3).

However, whether or not the enhancement of current in this channel with enhanced conductivity is possible will depend on whether the magnetospheric source is able to supply field-aligned currents this peculiar "discharge gap", as shown in Fig.6.

Fig. 5 shows the picture of field-aligned currents that is "offered" by the magnetosphere. One can see that "demand" and "supply" are more or less identical for the arrangements of the zones. It should be noted that the integral over all inflow and outflow currents in Fig. 5 is virtually zero.

\section{Conclusion}

The results presented in this study induce us to hope that the compatibility of field-aligned currents of magnetospheric and ionospheric origins is feasible.

Perhaps the mystery of the substorm lies in the plasma pressure distribution, or more precisely, in the global redistribution of the plasma pressure on the night side of the magnetosphere. Direct observations of plasma distribution in the magnetosphere are faced with large difficulties, because pressure must be known everywhere in the plasma sheet at high resolution, which in situ satellites have been unable to provide. Modelling of distribution of plasma pressure (on $\sim 3-12 \mathrm{R}_{\mathrm{E}}$ ) is very important, because the data from multisatellite magnetospheric missions for these purposes would be a very expensive project. Therefore there arises a necessity for modelling processes of near-Earth space. A selection and applying of correct initial system of equations are also very important. 


\section{References}

Ellis, P., Southwood D. J.: 1983. Reflection of Alfven waves by non-uniform ionospheres. Planet. Space Sci., 31, 107-117.

lijima, T., Potemra, T.A.: 1978. Large-scale characteristics of field-aligned currents associated with substorms. J. Geophys. Res., 83, P. 599-615.

Heppner J. P.: 1977. Empirical Models of High-Latitude Electric Fields. J. Geophys. Res. 82 (7), 1115-1125.

Kennel, C.F.: 1969. Consequence of a magnetospheric plasma. Rev. Geophys., 7, N 1-2, 379-419.

Ponomarev, E.A., 1985. Mechanism of magnetospheric substorms. Moscow: Nauka., pp 157, (in Russian).

Ponomarev, E.A., Sedykh, P.A.: 2006. How can we solve the problem of substorms? (Review). Geomagnetism and aeronomy. Pleiades Publishing Inc., 46, N4, 560-575.

Ponomarev, E.A., Sedykh, P.A., Urbanovich, V.D.: 2006. Generation of electric field in the magnetosphere, caused by processes in the bow shock. J. Atmos. and Sol. Terr. Phys. Elsevier Science, 68, 679-684.

Sedykh, P.A., Ponomarev, E.A.: 2002. The magnetosphereionosphere coupling in the region of auroral electrojets. Geomagnetism and aeronomy. Pleiades Publishing Inc., 42, N5, 613-618.

Sedykh, P.A.: 2011. On the role of the bow shock in power of magnetospheric disturbances. Sun \& Geosphere. Special issue of ISWI /United Nations/NASA/JAXA/AGU/ESA/; ISSN 1819-0839. 6(1), P.27-31.

Sedykh, P.A., Ponomarev, E.A.: 2012. A structurally adequate model of the geomagnetosphere. Stud. Geophys. Geod., AS CR, Prague, Inst.Geophys. V. 56, DOI: 10.1007/s11200-0119027-3, P. 110-126.

Sedykh, P.A.: 2014. Bow shock. Advances in Space Research, Elsevier Science. DOI:10.1016/j.asr.2014.03.015, JASR11746.

Vasyliunas, V. M.: 1970. Mathematical models of magnetospheric convection and its coupling to the ionosphere, Particles and Fields in the Magnetosphere, ed. B.M. McCormac, Higham, Mass., Holland, P. 60-71.

Volland H.: 1975. Models of Global Electric fields within the magnetosphere. Ann. Geophys.. 31. - P.159.

Yoshikawa, A., O. Amm, H. Vanhamäki, Fujii R.: 2013. Illustration of Cowling channel coupling to the shear Alfven wave, J. Geophys. Res. Space Physics, 118, 6405-6415, doi:10.1002/jgra.50513.(a)

Yoshikawa, A., O. Amm, H. Vanhama“ ki, A. Nakamizo, Fujii R.: 2013. Theory of Cowling channel formation by reflection of shear Alfven waves from the auroral ionosphere, J. Geophys. Res. Space Physics, 118, 6416-6425, doi:10.1002/jgra.50514.(b).

Zhu, L., J. J. Sojka, R. W. Schunk, Crain D. J.: 1993. Influence of horizontal inhomogeneity in the ionosphere on the reflection of Alfven waves. Geophys. Res. Lett., 20(4), 313-316, doi:10.1029/93GL00079. 